\title{
SYNCHRONOUS MACHINE PARAMETERS FROM SUDDEN-SHORT TESTS BY BACK-SOLVING
}

\author{
Fernando L. Alvarado, Senior Member \\ The University of Wisconsin
}

Claudio Cañizares

The University of Wisconsin

\section{ABSTRACT}

Calculation of synchronous machine parameters from sudden short circuit measurements has been subject to unneccssary assumptions and approximations. These assumptions are due in part to the unavailability of measurements, and in part to the cumbersome nature of the calculations needed to remove these approximations. This paper describes the nature of these approximations and a procedure to remove them. It also illustrates how a new philosophy for problem formulation and solution using backsolving programs can simplify and clarify the solution process. Finally, the paper introduces a new way of calculating cigenvalues based on a generalization of the eigenvalue problem. KEYWORDS: Synchronous Machines, Short Circuit Tests, Machine Parameters, Symbolic Computation.

\section{INTRODUCTION}

The calculation of synchronous machine parameters from sudden short circuit measurements has been subject to onc unnecessary assumption and two approximations for mathematical convenience.

- The first approximation involves neglecting the coupling between transient and subtransient periods. Considering that the time constants associated with the transient and subtransient periods are independent of each other, simple formulas for the calculation of machine inductances are possible. Considering that these time constants are not independent but represent cigenvalues of the natural response of the machine under sudden short circuit conditions, coupling between these time constants can be accounted for. Derivation of these formulas requires an analytic solution of a two by two eigenvalue problem. This work was done by Dommel et al. [1,2,3] as an extension to Canay [4].

- The second approximation involves neglecting armature resistance. If the armature resistance is considered, coupling occurs between the direct and quadrature parameters of the machine. It is still possible to view the time constants as eigenvalues, but all four cigenvalues are coupled. An analytic solution is ncarly impossible with conventional tools.

- The assumption is the result of the measurements normally available from tests not being sufficient to determine all the machine inductances. A new test proposed by Canay resolves this problem.

This paper describes a new approach to formulation of the problem that relies on the use of SOLVER-Q, a symbolically assisted numeric equation solving program $[8,9]$

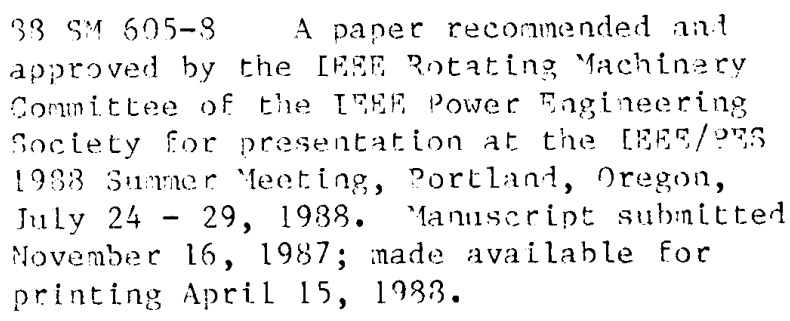

to calculate the machine parameters. With this program, no approximations are needed. An exact solution to the problem is obtained directly from its formulation.

Results of calculations for typical machine parameters indicate that the usual approximations are quite good for typical ranges of values of machine parameters. With this new tool no approximations are necessary. This improves the accuracy of machine models for typical machines and allows the extension of the theory to unusual design machines. Furthermore, because the solution is obtained directly from the defining formulas of the machine without further manipulations, additional physical insight into the meaning of all machine parameters can be gained without the need to become deeply involved in unnecessary mathematical manipulations.

\section{NOTATION}

We do not describe nuances of units and scaling associated with machine parameters. We use an accepted formulation of the machine equations using a more or less standard notation. A slightly different notation is required by the back-solver. Table 1 illustrates the notation for main variables. Table 2 illustrates the notation for subscripts. Mutual inductances have a single subscript whenever this is sufficient to avoid confusion.

Table 1: Notation for variables.

\begin{tabular}{|r|c|c|}
\hline Variable & In Equation & In SOLVER-Q \\
\hline Flux Linkage & $\lambda$ & 1 \\
Current & $i$ & $i$ \\
Voltage & $V$ & $V$ \\
Self Inductance & $L$ & $x$ \\
Mutual & $M$ & $\times \mathrm{m}$ \\
Resistance & $\mathrm{R}$ & $\mathrm{r}$ \\
Eigenvalues & $\mu$ & $\mu$ \\
Time Constants & $\mathrm{T}$ & $\mathrm{t}$ \\
\hline
\end{tabular}

Table 2: Notation for subscripts.

\begin{tabular}{|r|c|c|}
\hline Subscript & In Equations & SOLVER-Q \\
\hline Radian Frequency & $w$ & $w$ \\
Direct Axis Quantities & $d$ & $d$ \\
Firect Axis Armature Reaction & $d$ & $d$ \\
Field Winding & $f$ & $f$ \\
Direct Axis Damper Winding & 0 & $d p$ \\
Quadrature Axis Quantities & $q$ & $q$ \\
Quad. Axis Armature Reaction & $q$ & $q$ \\
Quad. Axis Damper Winding & 0 & $q p$ \\
Quad. Axis 2nd Winding & $g$ & $g$ \\
Open Circuit & 0 & 0 \\
Leakage & $\ell$ & 1 \\
\hline
\end{tabular}

\section{BACKGROUND}

The fundamental normalized equations used to describe the transient behavior of the machine are those for the ideal synchronous machine as defined in [13, page 700]:

$$
\begin{aligned}
& {\left[\begin{array}{l}
\lambda_{d} \\
\lambda_{f} \\
\lambda_{D}
\end{array}\right]=\left[\begin{array}{lll}
L_{d d} & M_{d} & M_{d} \\
M_{d} & L_{f f} & M_{d} \\
M_{d} & M_{d} & L_{D D}
\end{array}\right]\left[\begin{array}{c}
i_{d} \\
i_{f} \\
i_{D}
\end{array}\right]} \\
& {\left[\begin{array}{l}
\frac{d \lambda_{f}}{d t} \\
\frac{d \lambda_{D}}{d t}
\end{array}\right]=\left[\begin{array}{ll}
R_{f} & 0 \\
0 & R_{D}
\end{array}\right]\left[\begin{array}{l}
i_{f} \\
i_{D}
\end{array}\right]+\left[\begin{array}{l}
v_{f} \\
0
\end{array}\right]}
\end{aligned}
$$




$$
\begin{aligned}
& {\left[\begin{array}{l}
\lambda_{q} \\
\lambda_{g} \\
\lambda_{Q}
\end{array}\right]=\left[\begin{array}{ccc}
L_{q q} & M_{q} & M_{q} \\
M_{q} & L_{g g} & M_{q} \\
M_{q} & M_{q} & L_{Q Q}
\end{array}\right]\left[\begin{array}{c}
i_{q} \\
i_{g} \\
i_{Q}
\end{array}\right]} \\
& -\left[\begin{array}{c}
\frac{d \lambda_{q}}{d t} \\
\frac{d \lambda_{Q}}{d t}
\end{array}\right]=\left[\begin{array}{cc}
R_{g} & 0 \\
0 & R_{Q}
\end{array}\right]\left[\begin{array}{c}
i_{g} \\
i_{Q}
\end{array}\right]
\end{aligned}
$$

These equations ignore armature resistance. As a result, the direct and quadrature axis of the machine are decoupled and can be dealt with separately. Since the calculation procedures are the same for both axis, we describe them only for one axis.

The parameters of the machine can be determined from sudden short circuit tests prescribed by standards $[5,6]$. The measurements in Table 3 are available. Some of the normalized machine parameters above can be obtained directly from the test parameters in Table 3:

Table 3: Measurements Available.

\begin{tabular}{|r|l|}
\hline Measurement & Symbol \\
\hline Armature resistance & $R_{a}$ \\
Armature leakage reactance & $x_{l}$ \\
Zero sequence reactance & $x_{0}$ \\
Synchronous reactances & $x_{d}, x_{q}$ \\
Transient reactances & $x_{d}^{\prime}, x_{q}^{\prime}$ \\
Subtransient reactances & $x_{d}^{\prime \prime}, x_{a}^{\prime \prime}$ \\
Transient short cct time const. & $T_{d}^{\prime}, T_{q}^{a}$ \\
Subtransient short cct time const. & $T_{d,}^{\prime \prime}, T_{a}^{\prime \prime}$ \\
\hline
\end{tabular}

$$
\begin{array}{ll}
L_{d d}=\frac{x_{d}}{\omega} & M_{d}=\frac{x_{d}-x_{l}}{\omega} \\
L_{q q}=\frac{x_{d}}{\omega} & M_{q}=\frac{x_{q}-x_{l}}{\omega}
\end{array}
$$

In order to find the remaining direct axis parameters, first define the open circuit time constants. The traditional (approximate) formulas relating the open circuit and short circuit time constants are [7]:

$$
T_{d o}^{\prime}=\frac{x_{d}}{x_{d}^{\prime}} T_{d}^{\prime} \quad T_{d o}^{\prime \prime}=\frac{x_{d}^{\prime}}{x_{d}^{\prime \prime}} T_{d}^{\prime \prime}
$$

The traditional formulas for calculation of the remaining internal parameters assume that the transient and subtransient time constants are sufficiently different that the flux decays do not interact. These formulas are:

$$
\begin{gathered}
\frac{1}{x_{f f}}=\frac{1}{x_{d}^{\prime}-x_{l}}-\frac{1}{x_{M d}} \\
\frac{1}{x_{D D}}=\frac{1}{x_{d}^{\prime \prime}-x_{l}}-\frac{1}{x_{d}^{\prime}-x_{l}} \\
R_{f}=\frac{x_{f f}+x_{M d}}{\omega T_{d o}} \\
R_{D}=\frac{x_{D D} x_{M d}+x_{D D} x_{f f}+x_{f f} x_{M d}}{\omega T_{d o}^{\prime \prime}\left(x_{f f}+x_{M d}\right)}
\end{gathered}
$$

Similar equations apply to the quadrature axis.

\section{NEW IDEAS FOR EIGENVALUE COMPUTATIONS}

A back solving (or declarative style) program is a program capable of solving consistent sets of algebraic equations [12]. The philosophy of this paper is to establish a consistent set of equations, no matter how obtained, and to solve them as they are without further alteration. This paper uses SOLVER-Q [8,9], a declarative style equation handler that solves sets of nonlinear equations of arbitrary form. Thus, rather than obtaining explicit expressions for the machine parameters in terms of available measurements, we are satisfied with a set of consistent inverse expressions for measurements in terms of machine parameters. We then rely on the back solving ability of SOLVER-Q to perform the numerical computation. The desired parameters are uniquely but implicitly defined.

The relationship between parameters and measurements requires the solution of an eigenvalue problem. It is of interest to see how to express an eigenvalue problem for direct solution by back solving methods, so we digress slightly from our main topic of interest.

\section{Eigenvalues by Back Solving Methods}

Consider first the classical eigenvalue and eigenvector problem. The problem arises in the context of the solution of dynamic equations of the form:

$$
\frac{d y}{d t}=A y+u
$$

where $y$ is a state vector and $u$ is a forcing function.

The natural modes of response of this system are determined by the eigenvalues of this system. The eigenvalues and eigenvectors of this system are values of the scalar $\mu$ and the vector $y$ that satisfy:

$$
A y=\mu y
$$

The problem is to find a nonzero value for $y$ plus a value for $\mu$. If $A$ is a matrix of dimension $n$, it is known that in general there will be up to $n$ distinct eigenvalues and eigenvectors. Considering that the coupling between modes has been entirely ignored in most calculations to date, we do not incur a serious error if we limit this discussion to the case of distinct real eigenvalues.

In the definition above, there are $n+1$ variables (the $n$ components of $y$ and $\mu$ ), but only $n$ nonlinear equations. An additional equation is required to insure that the eigenvectors are not zero. There are many ways of establishing this condition, but the simplest two are to either set the norm of the eigenvector $y$ to unity, or to arbitrarily designate a single element of the eigenvector $y$ as one. Thus, the additional equations are either:

$$
\sum_{i=1}^{n} y_{i}^{2}=1 \quad \text { or } \quad y_{1}=1
$$

This latter approach can sometimes lead to problems, but is more expedient. Thus, the philosophy of reducing eigenvalue problems to ordinary nonlinear algebraic equation problems results in the need to solve:

$$
\begin{gathered}
a_{11} y_{1}+a_{12} y_{2}+\ldots=\mu y_{1} \\
a_{21} y_{2}+a_{22} y_{2}+\ldots=\mu y_{2} ; \\
\ldots \\
y_{1}=1 ;
\end{gathered}
$$

Other solution techniques to solve the eigenvalue problem above are described in references such as [10]. Here we need a more general view of what is an eigenvalue problem. Consider the differential equation:

$$
B \frac{d y}{d t}=A y+u
$$

The eigenvalues and eigenvectors are the solution of the following set of equations:

$$
A y=\mu B y
$$

$B$ must be nonsingular. In theory, this problem is reducible to the former problem as follows:

$$
J y=\mu y \quad \text { where: } \quad J=B^{-1} A
$$


There are, however, both computational and physical rcasoning advantages for leaving the equations as originally stated in (10). Sparsity, symmetry and insight are lost. The cigcnvalue problem can be formulated directly from (10) as the solution of the following set of nonlinear equations:

$$
\begin{gathered}
a_{11} y_{1}+a_{12} y_{2}+\ldots=\mu b_{11} y_{1}+\mu b_{12} y_{2}+\ldots ; \\
a_{21} y_{2}+a_{22} y_{2}+\ldots= \\
=b_{21} y_{1}+\mu b_{22} y_{2}+\ldots ; \\
\ldots \\
y_{1}=1 ;
\end{gathered}
$$

The next step in the generalization of the eigenvalue problem is to recognize that a set of linear equality constraints among dynamic variables can be considered in much the same way. In order for this to be possible in general, the differential equation must depend on additional variables $y$ that are linearly related to the state variable $x$ according to the following formulation:

$$
\begin{gathered}
K \frac{d y}{d t}=A y+B x+u \\
C y+D x=v
\end{gathered}
$$

If $D$ and $k$ are nonsingular, this problem can also be reduced to a standard eigenvalue problem as follows:

$$
J y=\mu y \quad \text { where: } \quad J=K^{-1}\left(A-B D^{-1} C\right) x
$$

The next step in the generalization of the problem is to recognize that not all variables may appear in the original differential equations. There may be additional variables introduced as part of the formulation process. We refer to the vector of these variables as $z$. Consider the following dynamic problem:

$$
\begin{aligned}
& K \frac{d y}{d t}=A y+B x+U \\
& C y+D x+E z=v \\
& F y+G x+H z=0
\end{aligned}
$$

where both $u$ and $v$ are forcing functions and $A, B, C, D$, $E, F, G$ and $K$ are matrices. $E, K$ and $\left(G-H E^{-1} D\right)$ are nonsingular. The state variables $y$ now participate not only in the differential equations, but are subject to additional equality constraints. Additional variables $Z$ are introduced, one per additional equality constraint. The eigenvalue problem associated with this system can be expressed as:

$$
\begin{gathered}
A y+B x=\mu K y \\
C y+D x+E z=0 \\
F y+G x+H z=0
\end{gathered}
$$

This problem is reducible to an ordinary eigenvalue problem as follows:

$$
\begin{gathered}
J y=\mu y \\
\text { where: } J \stackrel{\Delta}{=} K^{-1}\left[A+B\left(G-H E^{-1} D\right)^{-1}\left(H E^{-1} C-F\right)\right]
\end{gathered}
$$

Often it is easier to solve the eigenvalue problem using the complete set of equations in $(15)$ than the reduced set in (17). The solution of (16) is more direct and simpler due to the loss of symmetry and sparsity in $\mathrm{J}$.

In the problem of machine parameter determination from terminal measurements, we face a question opposite to the cigenvalue problem: we are given the eigenvalues, and we wish to find the values of the parameters that are necessary to produce the observed behavior. In the equations above, it is as if we were given the values of the $\mu$ 's and were asked to obtain the values of some of the matrix cocfficients. For every eigenvalue known, one coefficient can be obtained.

\section{Eigenvectors and Initial Conditions}

Solving the eigenvalue and eigenvector problems simplifies the solution of the complete problem including constant forcing terms $u$ and $v$. Assume that all $n$ eigenvalues $\mu_{i}$ and eigenvectors $y^{i}$ have been found and that are all distinct. Assume further that initial conditions for the state variables $y^{O}$ are available. The complete time domain response of this system can be determined from:

$$
\begin{aligned}
& y(t)=\alpha_{1} y^{1} e^{\mu 1} t+\alpha_{2} y^{2} e^{\mu 2^{t}}+\ldots+y^{p}(t) \\
& z(t)=\alpha_{1} z^{1} e^{\mu 1} t+\alpha_{2} z^{2} e^{\mu 2^{t}}+\ldots+z^{p}(t)
\end{aligned}
$$

where $y^{p}(t)$ and $z^{p}(t)$ are the forced response of the system, determined from:

$$
\begin{gathered}
A y^{p}+B x^{p}+u=0 \\
C y^{p}+D x^{p}+E z^{p}=v \\
F y^{p}+G x^{p}+H z^{p}=0
\end{gathered}
$$

The values of $\alpha_{1}, \alpha_{2}$, are calculated from:

$$
y(0)=\alpha_{1} y^{1}+\alpha_{2} y^{2}+\ldots+y^{P}(0)
$$

\section{FIRST STEP: DECOUPLED D AND Q AXIS}

Removing the approximations of the classic methods requires eliminating the assumption of independence between the transient and subtransient periods. We first neglect armature resistance, decoupling the $d$ and $q$ axis. This part of the paper parallels the work of Canay and Dommel, although the method used to solve the problem is quite different and sets the stage for the following sections.

In the problem of machine parameter determination, the objective is to obtain the values of some of the equation parameters. Using the back solving philosophy, both the formulation and the final form for the problem are the same.

The equations that determine the dynamic behavior of the direct axis of the machine are:

$$
\begin{aligned}
\lambda_{d}=L_{d d} i_{d}+M_{d} i_{f}+M_{d} i_{D} \\
\lambda_{f}=M_{d} i_{d}+L_{f f} i_{f}+M_{d} i_{D} \\
\lambda_{D}=M_{d} i_{d}+M_{d} i_{f}+L_{D D} i_{D} \\
-\frac{d \lambda_{f}}{d t}=R_{f} i_{f}+v_{f} \\
-\frac{d \lambda_{D}}{d t}=R_{D} i_{D}
\end{aligned}
$$

These equations fit the form of the generalized eigenvalue problem with linear equality constraints. Two alternatives for solution exist. The first is to obtain an explicit systematic procedure for the exact calculation of eigenvalues and eigenvectors as done by Canay. For convenience, this procedure assumes the use of unsaturated open circuit time constants for the machine obtained from:

$$
\begin{aligned}
& T_{d o}^{\prime}+T_{d o}^{\prime \prime}= \frac{x_{d}}{x_{d}^{\prime}} T_{d}^{\prime}+\left(1-\frac{x_{d}}{x_{d}^{\prime}}+\frac{x_{d}}{x_{d}^{\prime \prime}}\right) T_{d}^{\prime \prime} \\
& T_{d o}^{\prime} T_{d o}^{\prime \prime}=T_{d}^{\prime} T_{d}^{\prime \prime} \frac{x_{d}}{x_{d}^{\prime \prime}}
\end{aligned}
$$

The remainder of the Canay procedure is outlined in Appendix A. The Canay procedure determines the unknown machine parameters $R_{f}, R_{D}, L_{f f}$ and $L D D$. The procedure is simple and direct but has some shortcomings:

- The original formulas require considerable modification, obscuring insight.

- Explicit formulas can only be obtained for systems of low order, 2 in Canay's case. Analytic expressions for high order systems are impossible.

- Slight variations in available information can cause havoc. For example, if $R_{f}$ happens to be known instead of $X_{d}^{\prime}$.

The back solving procedure requires that we formulate the problem as above, in a consistent unambiguous manner, 
and then use a back solver to obtain results. This approach can accommodate to any manner of measurements and specifications with ease. The formulation for the solution of the machine problem considering that $R_{a}=0$ consists of several kinds of equations assembled as one set. Each equation kind is described separately, but in the end they are all solved simultaneously.

\section{a) Eigenvalue/eigenvector equations:}

These equations describe the natural response modes of the system. The eigenvalue equations for the machine consist of two sets of 5 equations, one for each eigenvalue. The set of equations that define in implicit form the eigenvalues is:

$$
\begin{gathered}
-\mu_{i} \lambda_{f i}=R_{f} i_{f i} \\
-\mu_{1} \lambda_{D i}=R_{D} i_{D i} \\
0=L_{d d} i_{d i}+M_{d} i_{f i}+M_{d} I_{D I} \\
\lambda_{d i}=M_{d} i_{d i}+L_{f f} i_{f i}+M_{d} I_{D I} \\
\lambda_{D i}=M_{d} i_{d i}+M_{d} i_{f i}+L_{D D} I_{D i}
\end{gathered}
$$

To obtain the first set, let $i=1$. To obtain the second set, let $i=2$. The forcing functions $v_{f}$ and $\lambda_{d}$ are removed from the natural response determination. They can be found by solving a set of steady state equations.

b) Equations for $\mu_{1}$ and $\mu_{2}$ from measurements:

Our problem is a special case of the generalized eigenvalue problem, where the vector $z$ is now the scalar $i_{d}$. The solution to this problem has the form:

$$
i_{d}(t)=i_{d}^{p}(t)+\alpha_{1} i_{d 1} e^{\mu / t}+\alpha_{2} i_{d 2} e^{\mu 2 t}
$$

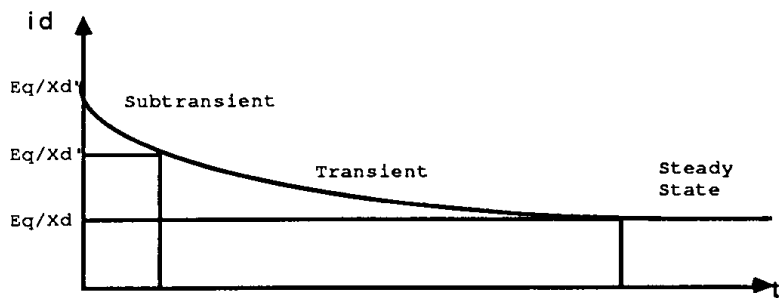

Figure 1: The determination of time constants and reactances from measurements.

According to standards, the short circuit current is defined as:

$$
i_{d}=\frac{E_{q}}{x_{d}}+E_{q}\left(\frac{1}{x_{d}^{\prime}}-\frac{1}{x_{d}}\right) e^{-t / T_{d}^{\prime}}+E_{q}\left(\frac{1}{x_{d}^{\prime \prime}}-\frac{1}{x_{d}^{\prime}}\right) e^{-t / T^{\prime}}
$$

From these equations we conclude that the values of the eigenvalues $\mu$ and the scaling coefficients $\alpha$ are known in terms of some of the (still unknown) parameters. Thus, we establish some additional equations:

$$
\begin{array}{cc}
\mu_{1}=-\frac{1}{T_{d}^{\prime}} & \mu_{2}=-\frac{1}{T_{d}^{\prime \prime}} \\
\alpha_{1} i_{d 1}=E_{q}\left(\frac{1}{x_{d}^{\prime}}-\frac{1}{x_{d}}\right) \quad \alpha_{2} I_{d 2}=E_{q}\left(\frac{1}{x_{d}^{\prime \prime}}-\frac{1}{x_{d}^{\prime}}\right)
\end{array}
$$

\section{c) Initial condition and steady state equations:}

$\alpha_{1}, \alpha_{2}$ and $E_{d}$ are calculated based on initial and final conditions. The initial condition equations are determined from the state variables $\lambda_{f}$ and $\lambda_{D}$ before the sudden short circuit. If the machine is operating under open circuit nominal conditions prior to the fault, the following equations can be established:

$$
\begin{gathered}
\lambda_{d O}-=\frac{1}{\omega} \\
R_{f} i_{f O}+v_{f}=0 \\
\lambda_{d O}=M_{d} l_{f O}-
\end{gathered}
$$

$$
\begin{aligned}
& \lambda_{f O}=L_{f f} i_{f O}- \\
& \lambda_{D O}=M_{d} l_{f O}-
\end{aligned}
$$

The equations for final values (the short circuit at steady state) are:

$$
\begin{gathered}
R_{f} l_{f p}+v_{f}=0 \\
0=L_{d d} I_{d p}+M_{d} i_{f p} \\
\lambda_{p p}=M_{d} i_{d p}+L_{f f} i_{f p} \\
\lambda_{D p}=M_{d}\left(i_{d p}+i_{f p}\right)
\end{gathered}
$$

Once we know these values, $\alpha_{1}, \alpha_{2}$ and $E_{q}$ can be calculated from the following equations:

$$
\begin{gathered}
\alpha_{1} \lambda_{11}+\alpha_{2} \lambda_{f 2}=\lambda_{f D}-\lambda_{f p} \\
\alpha_{1} \lambda_{D 1}+\alpha_{2} \lambda_{D 2}=\lambda_{D O}-\lambda_{D p} \\
E_{q}=-\omega \lambda_{d O}-
\end{gathered}
$$

Assembling equations $(23-29)$ is sufficient to solve the problem provided a reasonable initial guess is available. There is no need for an explicit analytic solution in the traditional sense. Appendix B describes the basis for this assertion. Reasonable initial guesses can be obtained by solving the approximate problem using traditional formulas.

\section{SECOND STEP: THE COUPLED EIGENVALUE PROBLEM}

If the armature resistance is not ignored, the problem of calculating the machine parameters from an eigenvalue formulation appears to become far more involved. However, the philosophy is identical to the one above, and in principle as well as practice, the problem is not much harder than before.

The eigenvalue/eigenvector equations to be solved include now the linkage fluxes for the $q$ and the $d$ axis; other equations neglect this interaction. This assumption is required because the classical definition of $i_{d}$ for the sudden short circuit test, since this current would depend on four time constants, not two. This does not agree with the data extracted from measured short circuit currents as illustrated in Figure 1. The numerical example shown below supports the validity of this slight approximation.

The equations to be solved are four sets of eigenvalue definition equations, one for each eigenvalue. Two eigenvalues correspond to the $d$ axis, two to the $q$ axis.

$$
\begin{aligned}
& -\mu_{1} \lambda_{d i}=R_{a} I_{d i}+\omega \lambda_{q i} \quad-\mu_{1} \lambda_{q i}=R_{a} I_{q i}-\omega \lambda_{d i} \\
& -\mu_{1} \lambda_{f i}=R_{f} I_{f i} \quad-\mu i \lambda_{g i}=R_{g} i_{g i} \\
& -\mu_{i} \lambda_{D i}=R_{D} I_{D I} \quad-\mu \mid \lambda_{Q i}=R_{Q} I_{Q} \\
& \lambda_{d i}=L_{d d} i_{d i}+M_{d} i_{f i}+M_{d} i_{D i} \quad \lambda_{q i}=L_{q q} i_{q i}+M_{q} i_{q i}+M_{q} i_{Q i} \\
& \lambda_{f i}=M_{d} I_{d i}+L_{f f} i_{f i}+M_{d} i_{D i} \quad \lambda_{q i}=L_{q q} i_{q i}+M_{q} i_{g i}+M_{q} i_{Q i} \\
& \lambda_{D i}=M_{d} i_{d i}+M_{d} l_{f i}+L_{D D} i_{D i} \quad \lambda_{Q i}=M_{q} i_{q i}+M_{q} I_{g i}+L_{Q Q} i_{Q i}
\end{aligned}
$$

Four sets of these equations are obtained by letting $i$ be $1,2,3$ and 4 , since there are four time constants for the $d$ and $q$ axis:

$$
\mu_{1}=-\frac{1}{T_{d}^{\prime}} \quad \mu_{2}=-\frac{1}{T_{d}^{\prime \prime}} \quad \mu_{3}=-\frac{1}{T_{q}^{\prime}} \quad \mu_{4}=-\frac{1}{T_{q}^{\prime \prime}}
$$

We now establish general initial condition equations. We do not require an open circuit initial condition during the sudden short circuit test.

$$
\begin{array}{cr}
\alpha_{1} i_{d 1}=E_{q}\left(\frac{1}{x_{d}^{\prime}}-\frac{1}{x_{d}}\right) & \alpha_{2} I_{d 2}=E_{q}\left(\frac{1}{x_{d}^{\prime \prime}}-\frac{1}{x_{d}^{\prime}}\right) \\
\beta_{1} i_{q 3}=-E_{d}\left(\frac{1}{x_{q}^{\prime}}-\frac{1}{x_{q}}\right) & \beta_{2} I_{q 4}=-E_{d}\left(\frac{1}{x_{q}^{\prime \prime}}-\frac{1}{x_{q}^{\prime}}\right) \\
E_{d}=+\omega \lambda_{q 0}- & E_{q}=-\omega \lambda_{d 0}-
\end{array}
$$

The initial conditions also give expressions in terms of the values of $\lambda_{f O}, \lambda_{D O}, \lambda_{g O}$, and $\lambda_{Q 0}$. Short circuit steady state equations (considering $i_{f} p=i_{f 0}$ ), allow us to calculate 
228

$\lambda_{f}^{p}, \lambda_{D}^{p}, \lambda_{q}^{p}$, and $\lambda_{Q}^{p}$. Both sets of flux linkage are used to determine another set of expressions for $\alpha_{1}, \alpha_{2}, \beta_{1}$, and $\beta_{2}$, as follows:

$$
\begin{aligned}
& \alpha_{1} \lambda_{f 1}+\alpha_{2} \lambda_{f 2}=\lambda_{f 0}-\lambda_{f p} \\
& \alpha_{1} \lambda_{D 1}+\alpha_{2} \lambda_{D 2}=\lambda_{D O}-\lambda_{D p} \\
& \beta_{1} \lambda_{g 3}+\beta_{2} \lambda_{g 4}=\lambda_{g 0}-\lambda_{g p} \\
& \beta_{1} \lambda_{03}+\beta_{2} \lambda_{04}=\lambda_{00}-\lambda_{0 p}
\end{aligned}
$$

The complete set of equations (30-33) gives enough conditions to calculate the eight parameters $\left(R_{f}, R_{D}, L_{f f}\right.$, and $L_{D O}$ for the direct axis and $R_{g}, R_{q}, L_{g g}$, and $L_{Q Q}$ for the quadrature axis).

\section{FINAL STEP: THE CANAY INDUCTANCE}

The final step in removing the shortcomings of traditional machine parameter calculation methods requires the definition and measurement of a new machine parameter, the Canay inductance. The Canay inductance is of consequence only for the direct axis.

One of the assumptions in ( 1 ) is that the mutual inductances between all windings on one axis are numerically equal. The implication of this assumption is that the instantaneous values of some of the winding currents may be in error, but their effect as seen from the armature is correct. When the winding is a damper winding this makes sense, as the winding in question is only fictitious. However, when the winding in question is the field winding, we may be interested in its actual currents. In this case the actual value of the mutual inductance between armature and field in the direct axis is of interest. However, there are simply not enough measurements provided in the traditional tests to permit the calculation of this inductance. Thus, it is necessary to resort to an additional measurement. This was proposed by Canay. We describe the calculation of machine inductances when the Canay inductance is available.

While Canay and others have insisted on the need for an equivalent circuit representation of the magnetic circuit, we argue that introducing the electric circuit equivalent of the magnetic circuit obscures and limits the problem. We deal directly with the magnetic circuit and its equations, without reference to an equivalent circuit.

The equations for the direct axis of the machine, before referring quantities to the armature, are:

$$
\begin{aligned}
& {\left[\begin{array}{l}
\lambda_{d} \\
\lambda_{f} \\
\lambda_{D}
\end{array}\right]=\left[\begin{array}{lll}
L_{d d} & M_{d f} & M_{d D} \\
M_{d f} & L_{f f} & M_{f D} \\
M_{d D} & M_{f D} & L_{D D}
\end{array}\right]\left[\begin{array}{l}
i_{d} \\
i_{f} \\
i_{D}
\end{array}\right]} \\
& -\left[\begin{array}{c}
\frac{d \lambda_{f}}{d t} \\
\frac{d \lambda_{D}}{d t}
\end{array}\right]=\left[\begin{array}{cc}
R_{f} & 0 \\
0 & R_{D}
\end{array}\right]\left[\begin{array}{l}
i_{f} \\
i_{D}
\end{array}\right]+\left[\begin{array}{l}
v_{f} \\
0
\end{array}\right]
\end{aligned}
$$

The leakage reactance $X_{\ell}$ obtained from measurements represents leakage flux between armature and rotor windings, the damper winding in particular. If we know the $\mathrm{mmf} i_{d}$ on the armature winding, the difference between the fluxes in the armature and the damper winding is:

$$
\lambda_{d}-\lambda_{D}=x_{l} i_{d}
$$

From equation (34), this quantity is also equal to:

$$
\lambda_{d}-\lambda_{D}=\left(L_{d d}-M_{d D}\right) i_{d}
$$

Therefore the leakage flux allows us to establish:

$$
M_{d D}=L_{d d}-x_{\ell}
$$

Assume that we also know the flux that links the field winding but fails to link the armature and the damper windings of the machine. Call this the Canay flux, and its reactance the Canay reactance $X_{C}$ (Also known as the field leakage reactance $\left.X_{f_{1}}\right)$. According to this definition:

$$
\begin{gathered}
\lambda_{f}-\lambda_{D}=X_{C} i_{f} \\
\lambda_{f}-\lambda_{D}=\left(L_{f}-M_{f D}\right) i_{f} \\
M_{f D}=L_{f f}-X_{C}
\end{gathered}
$$

Knowledge of these leakage fluxes from measurements allows us to find two additional parameters. We observe that there are three unknown mutual couplings. One additional condition is obtained as follows. Define:

$$
\begin{aligned}
& \lambda_{f}^{\prime}=k_{f} \lambda_{f} i_{f}^{\prime}=\frac{1}{k_{f}} i_{f} \\
& \lambda_{D}^{\prime}=k_{D} \lambda_{D} i_{D}^{\prime}=\frac{1}{k_{D}} i_{D}
\end{aligned}
$$

Substitution into equation (34) yields:

$$
\begin{aligned}
& {\left[\begin{array}{c}
\lambda_{d} \\
\lambda_{f} \\
\lambda_{D}^{\prime}
\end{array}\right]=\left[\begin{array}{ccc}
L_{d d} & k_{f} M_{d f} & k_{D} M_{d D} \\
k_{f} M_{d f} & k_{f}^{2} L_{f f} & k_{f} k_{D} M_{f D} \\
k_{D} M_{d D} & k_{f} k_{D} M_{f D} & k_{D}^{2} L_{D D}
\end{array}\right]\left[\begin{array}{c}
i_{d} \\
i_{f} \\
i_{D}
\end{array}\right]} \\
& -\left[\begin{array}{c}
\frac{d \lambda_{f}}{d t} \\
\frac{d \lambda_{D}}{d t}
\end{array}\right]=\left[\begin{array}{cc}
k_{f}^{2} R_{f} & 0 \\
0 & k_{D}^{2} R_{D}
\end{array}\right]\left[\begin{array}{c}
i f \\
i d
\end{array}\right]+\left[\begin{array}{c}
k_{f} v_{f} \\
0
\end{array}\right]
\end{aligned}
$$

The primes can be dropped. The values of $k_{f}$ and $k_{D}$ are chosen so that the equations above are reduced to the form in (1). In order for this to be the case we choose:

$$
k_{f}=\frac{M_{d D}}{M_{f D}} \quad k_{D}=\frac{M_{d f}}{M_{f D}}
$$

Because we do not care about the actual values of damper winding flux and current, we can arbitrarily select $K_{D}=1$, which implies $M_{f D}=M_{d f}$. This provides the needed added "measurement." If the Canay leakage inductance is not known, then we make an additional (albeit incorrect) assumption. We assume that $k_{f}=1$.

The method for measuring the Canay reactance is left to the original paper. For the interested reader, the suggested procedure is to obtain a trace of the field current amplitude during the sudden short circuit test. The ratio of the initial amplitude of the field current oscillations to the initial field current indirectly defines the Canay reactance. Exact computation of this reactance is not trivial, and provides another opportunity for use of the back solving methodology advocated in this paper.

\section{NUMERICAL EXAMPLES}

We illustrate the new method with a numerical example from [11] and variations. In most cases there is little difference between traditional methods of calculation and the new method. It is only as the values of the armature resistance increase that significant difference can be observed.

We first reproduce the values of the desired parameters calculated using both Canay's explicit method as outlined in Appendix $\mathrm{A}$ and two variations of the method presented in this paper: we consider first both the decoupled $d / q$ formulation (which implies $R_{a}=0$ ) and then the complete formulation, but with the armature resistance $R_{a}$ set equal to zero. Table 4 illustrates the data used and Table 5 shows the results. We obtain the same results with both the Canay approach and either of the SOLVER-Q approaches.

From the complete eigenvalue formulation we can study the effect of $R_{a}$ on any parameter. Figures 2-5 show how changes in $R_{a}$ affect the calculated parameters. Notice 
that both the field resistance and inductance are relatively insensitive to changes in $R_{a}$, while other parameters are quite sensitive to $R_{a}$ beyond a certain range. A slight dependency of $R_{g}$ and $X_{g}$ on the sudden short initial conditions was observed when $R_{a}$ was not zero, but is not shown.

Table 4: Data for example from [7].

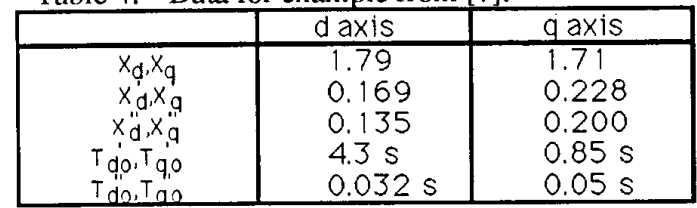

Table 5: Calculated Parameters neglecting $R_{a}$. Both methods give the same results.

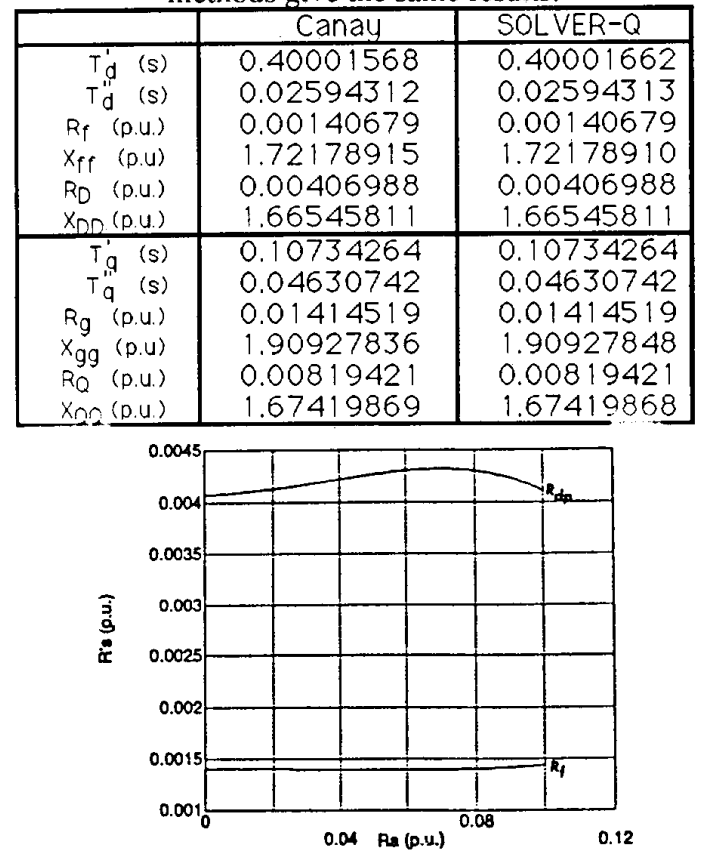

Figure 2: $R_{f}$ and $R_{d p}$ as a function of $R_{a}$. i he variation is slight. This assumes that $R_{f}$ was calculated, not measured.

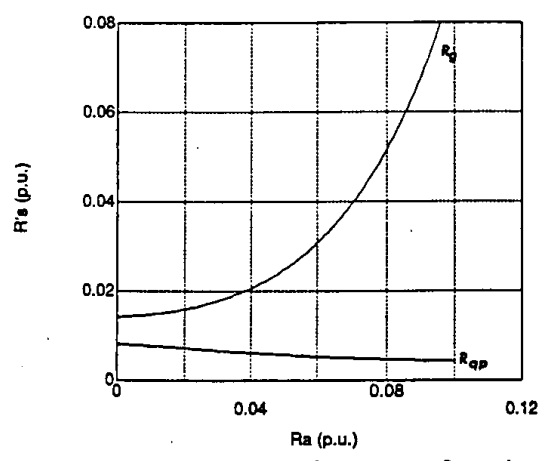

Figuri 3: Resistances $R_{g}$ and $R_{a p}$ as a function us $R_{a}$

Table 6 shows that the modal coupling between the $d$ and $q$ axis due the resistance $R_{a}$ is small. Even though the armature resistance results in interaction that affects the values of the own axis time constants themselves, the decay terms in either axis with the opposite axis time constants are small in amplitude.

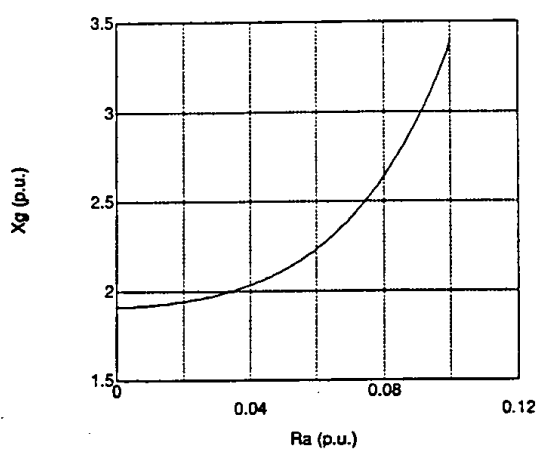

Figure 4: $X_{g}$ as a function of $R_{a}$

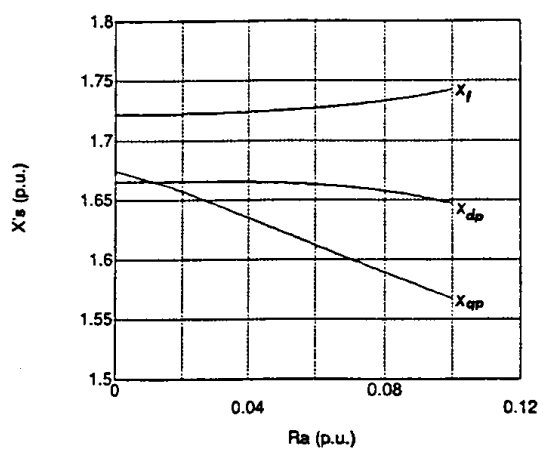

Figure 5: Other reactances as a function of $R_{a}$

Table 6: Selected eigenvector values for $\lambda_{f}$ calculated for the eigenvalues $T_{d}^{\prime}$ and $T_{q}^{\prime}$. A value of 0 for $\lambda_{\mathrm{f}} 3$ denotes no interaction between axis.

\begin{tabular}{|l|c|l|}
\hline$R_{a}$ (p.u.) & $\lambda_{f_{1}}\left(T_{d}\right)$ & $\lambda_{f 3}\left(T_{d}\right)$ \\
\hline 0 & 1.0 & 0 \\
0.00001 & 1.0 & 0.000014 \\
0.0001 & 1.0 & 0.000142 \\
0.001 & 1.0 & 0.001418 \\
0.01 & 1.0 & 0.014274 \\
0.05 & 1.0 & 0.068049 \\
0.1 & 1.0 & 0.112482 \\
\hline
\end{tabular}

\section{CONCLUSIONS}

The generalization of the eigenvalue problem along with the use of a back solver permits the removal of approximations during machine parameter determination. Approximations are unnecessary and the formulation is much cleaner than in previous efforts. Procedures that neglect $R_{a}$ in the calculation process (such as Canay's) introduce errors when time constants are re-calculated based on the parameters obtained (about $4 \%$ for $T_{a}$ ' with $R_{a}=0.04$ pu). The present procedure introduces no such errors. Even for large values of $R_{a}$ the time constants are correctly computed, since the coupling between axis is considered.

\section{ACKNOWLEDGEMENT}

The authors wish to thank the Electric Power Research Institute. The ideas upon which this work is based were developed as part of our work on instructional materials for EMTP use. In the process of attempting to describe how the EMTP performs the calculation of machine parameters we got to thinking about how it ought to be done in this era where sufficient computing power renders many approximations unnecessary. Also, special thanks are due to the reviewers of this paper for many valuable suggestions. 


\section{REFERENCES}

[1] H. W. Dommel, "Electromagnetic Transients Program Reference Manual (EMTP Theory Book)," Published by the Bonneville Power Administration, Portland, Oregon, August 1986.

[2] H. W. Dommel, "Data Conversion of Synchronous Machine Parameters," EMTP Newsletter, Vol. 1, No.3, pp. 13-17, April 1980.

[3] H. W. Dommel, S. Battacharya, I. I. Dommel, V. Brandwajn and Ye Zhong-liang, "Canay's Data Conversion of Synchronous Machine Parameters," EMTP Newsletter, Vol. 5, No. 4, pp. 8-25, October 1985.

[4] I. M. Canay, "Determination of Model Parameters of Synchronous Machines," Proceedings IEE, vol. 130, part B, pp. 86-94, March 1983.

[5] IEEE, "Test Procedures for Synchronous Machines," Standard 115, 1983.

[6] IEC, "Recommendations for Rotating Electric Machinery," Publication 34-4A, 1972.

[7] B. Adkins, The General Theory of Electrical Machines, Chapman-Hall 1957, London.

[8] F. L. Alvarado, "SOLVER-Q Instruction Manual," Software Development and Distribution Center, The University of Wisconsin, Madison, Wi, 1987.

[9] F. L. Alvarado and Y. Liu, "General Purpose Symbolic Simulation Tools for Electric Networks", Proceedings of IEEE PICA'87, May 1987, pp. 222-229.

[10] J. H. Wilkinson, "The Algebraic Eigenvalue Problem," Oxford University Press, London, 1965.

[11] IEEE Subsynchronous Resonance Task Force of the Dynamic System Performance Working Group, "First Benchmark Model for Computer Simulation of Subsynchronous Resonance," IEEE Transactions of Power Apparatus and Systems, Sept/Oct 1977.

[12] M. Konopasek and S. Jayaraman, "Constraint and Declarative Languages for Engineering Applications: The TK!Solver Contribution," Proceedings of the IEEE, Vol. 73, No. 12, December 1985.

[13] IEEE Standard Dictrionary of Electrical and Electronics Terms, $2^{\text {nd }}$ edition, Wiley-Interscience 1977.

\section{APPENDIX A: THE CANAY PROCEDURE}

The Canay procedure to calculate the parameters $R_{f}$, $R_{D}, L_{f f}$ and $L_{D D}$, of the synchronous machine is as follows:

a) Obtain the "f-branch" and "D-branch" time constants (some fictitious branches):

$$
\begin{array}{cc}
T_{1}=\frac{L_{f}}{R_{f}} & T_{2}=\frac{L_{D}}{R_{D}} \\
L_{f}=L_{f f}-M_{d} & L_{D}=L_{D D}-M_{d}
\end{array}
$$

These values are obtained as the solution of:

$$
\begin{gathered}
T_{1}+T_{2}=\left(T_{d_{0}}+T_{d_{0}}\right) \frac{M_{d}-L_{d}}{M_{d}}+\left(T_{d}^{\prime}+T_{d}^{\prime \prime}\right) \frac{L_{d}}{M_{d}} \\
T_{1} T_{2}=T_{d_{0}} T_{d_{0}}^{\prime \prime} \frac{L_{d f o}}{M_{d}}
\end{gathered}
$$

Where $L_{d f D}$ is found from the following equation:

$$
L_{d f D}=M_{d}-L_{d}+L_{d}
$$

b) Using the values of $T_{1}$ and $T_{2}$ calculated in (a), the inductance $L_{d f}$ can be obtained from:

$$
L_{d f}=\frac{M_{d}\left(T_{1}-T_{2}\right)}{T_{d_{0}}+T_{d o}-\left(1+\frac{M_{d}}{L_{d f D}}\right) T_{2}}
$$

c) Based on these parallel inductances $\left(L_{d f D}\right.$ and $\left.L_{d f}\right), L_{f}$ and $L_{D}$ can be calculated:

$$
L_{f}=\frac{L_{d f} M_{d}}{M_{d}-L_{d f}} \quad L_{D}=\frac{L_{d f D} L_{d f}}{L_{d f}-L_{d f D}}
$$

d) Knowing the values of $L_{f}$ and $L_{D}$, the desired machine parameters can be obtained from:
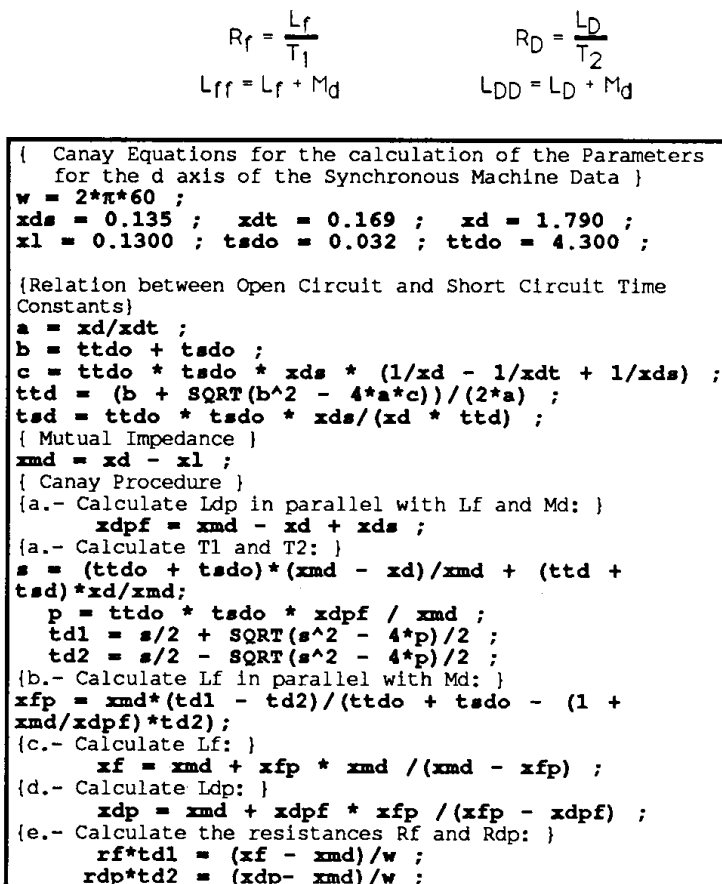

1 Canay Equations for the calculation of the Parameters for the $d$ axis of the Synchronous Machine Data \} $v=2 \star \pi \star 60$;

$x d=0.135 ; x d t=0.169 ; x d=1.790 ;$
$x 1=0.1300 ;$ tado $=0.032 ;$ tedo $=4.300 ;$

\{Relation between open Circuit and short Circult Time Constants\}

a $=\mathbf{x d} / \mathbf{x d t}$

b = ttdo + tado

c atdo t tsdo * xds * $(1 / x d-1 / x d t+1 / x d s)$.

ttd $=\left(b+3 Q R T\left(b^{\wedge} 2-4 t^{*} a\right)\right) /\left(2 \star_{a} a\right) ;$

tad = ttdo $*$ tado

I Mut ual Impedance 1

ind $=\mathbf{x d}-\mathbf{x l}$;

\{a.- Calculate Ldp in parallel with Lf and Md: \}

xdpf = xmd - xd + xds :

fa.- Calculate T1 and T2: \}

* = (ttdo + tedo) * (xmd - xd)/xend + (ttd +

tad) *xd/xind:

$p=$ ttdo * tedo * xdpf / xomd;

td1 $=/ 2+\operatorname{sQRT}\left(a^{\wedge} 2-4{ }^{*} \mathrm{P}\right) / 2 ;$

b. - Calculate Lf in parallel with Md:

$x f p=x^{\star}(t d 1-t d 2) /(t t d o+t$ ado $-(1+$ xmd (xdpf)*td2) :

(c. - Calculate If:

xf $=$ xnd $+x f_{p}$ * xnd $/\left(\right.$ and $\left.-x f_{p}\right)$;

Id. - Calculate Idp: 1

xdp $=$ xnd $+x d p f * x f_{p} /\left(x f_{p}-x d p f\right)$;

Calculate the resistances $R$

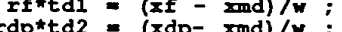

Figure B-1: SOLVER-Q file for the Canay procedure. Refer to Tables 1 and 2 for notation.

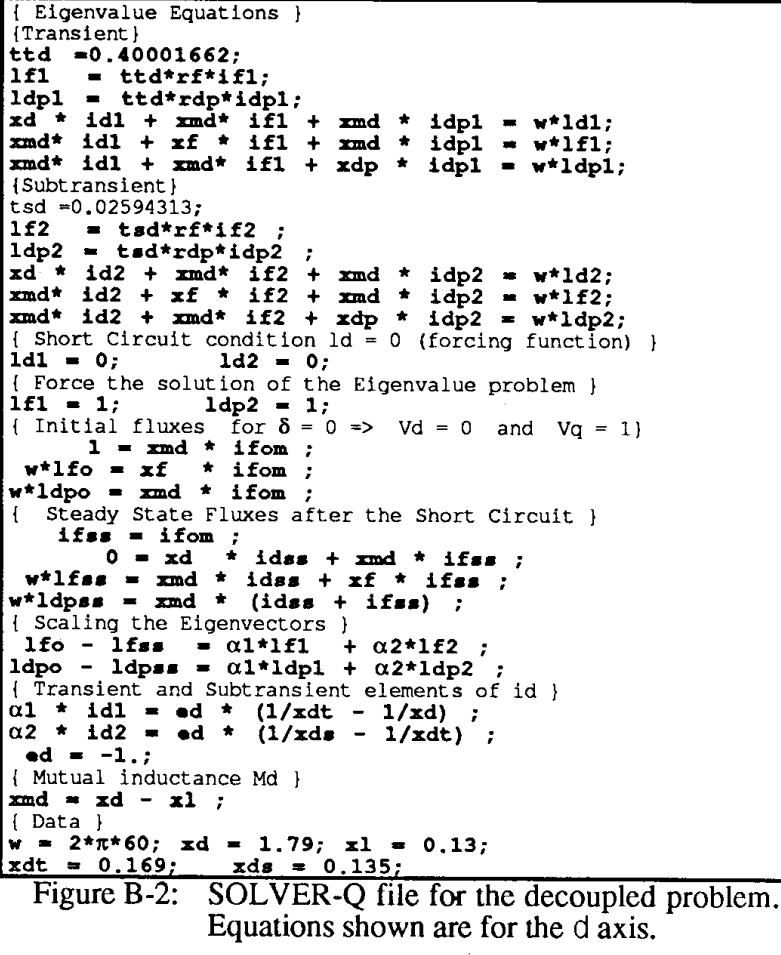




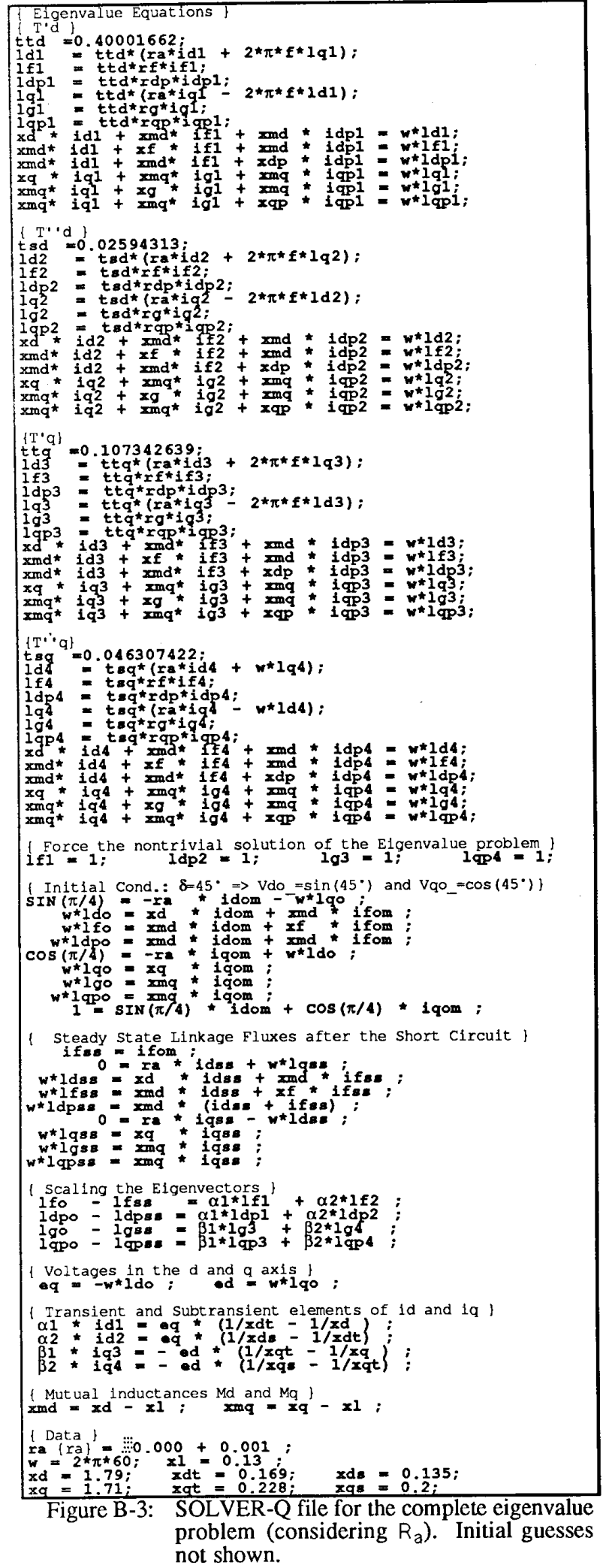

\section{APPENDIX B: SOLVER-Q SETUP}

The present work has been rendered practical only because of the recent introduction of a powerful symbolically assisted numeric equation solving package capable of manipulating large sets of coupled nonlinear equations with ease. This package, named SOLVER-Q, allows the direct transition from a well posed problem formulation to its numerical solution. This program is designed to operate in an IBM PC environment, where it can handle up to 1000 simultaneous nonlinear equations.

Here we present the complete setup in SOLVER-Q format for the example used in this paper. Notice that because of the limited font set on the IBM computer and because of SOLVER-Q's inability to distinguish lowercase from uppercase in variable names, some of the symbols have been changed. Specifically, we use 1 instead of $\lambda$ for flux and the subscript dp instead of $D$ to denote direct axis damper winding quantities.

SOLVER-Q can solve the problem starting for any consistent set of equations. It converges better if a reasonable initial guess is given. A reasonable initial guess for all the variables is the approximate solution obtained from the traditional formulas.

Fernando L. Alvarado (M'69 $\left.\mathrm{SM}^{\prime} 78\right)$ was born in Lima, Peru in 1945 . He received the BEE and PE degrees from the National University of Engineering in Lima, Peru, the MS degree from Clarkson College (now Clarkson University) in Potsdam, New York, and the Ph.D. degree from the University of Michigan in 1972. Dr. Alvarado joined the University of Toledo-Ohio, as an assistant professor in 1972. Since 1975 he has been with the University of Wisconsin in Madison, where he is currently a Professor in the Department of Electrical and Computer Engineering. Dr. Alvarado has authored numerous papers in the area of computer applications to engineering in general and power systems in particular. His main interests are in the area of large scale nonlinear networks, power system economics and control, symbolic computation, computing paradigms and robotic control. Dr. Alvarado is a member of CIGRE, Sigma Xi and ACM.

Claudio A. Cañizares was born in Mexico D.F. in 1960. He received his BSEE from the National Politechnique Institute (EPN) in Quito, Ecuador in April 1984, where he has been working since August 1983. Mr. Cañizares holds the position of Assistant Professor at this institution. Since August 1986 he

has been on leave from EPN working toward his MSEE and $\mathrm{Ph} . \mathrm{D}$. degrees at the University of Wisconsin-Madison, under a Fulbright Scholarship. He is colaborating with Dr. Fernando Alvarado as a research assistant, and working in the areas of symbolic computation applied to Power Systems and transient analysis of electric networks by means of the EMTP. 


\section{Discussion}

H.W. Dommel ('rhe Universjty of brjtish Co Iumbia, Vincouver, Candeda) and I.I. Dommel (Fower syisterit; Consultants, Vancouver, Canda.1): The duthor: presient a rovej and userul approach for the conversion of syochronous masthine: parameters, which takes the stator resistance $R_{\text {a }}$ accuratedy into account. For typical data, the improved accouracy over Canay': data converioion may be insignificant, lut an accurate solution is; always

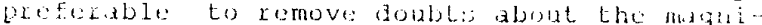
tude of deproximation errors. The athor:; method ia therefore a valuable alvance in the alata conversion problem.

We have ased the system of 6 differential equations for a sudlen three-nhase bhort-ciscuit a: the author: did, not for datid comversicon, but to cheres how well the: reaistances and inductances from various data conversion procertures reproduce the transient an! subtransient reactances and time: constants. While carrying out this work, we discovered that our data conversion of [1] wasi :ilightly inaccurate.

By diagonalizing the 6 differential equatione with the eigervalue/eigenvector approach, it is possible to write Gown the ionution expliaitly in the furm of Eu. (25). The tinie constiant:s are the regat ive recipsu ade of the eigenvalues, and the coefficients $\left(1 / X^{\prime} \mathrm{d} \cdots 1 / \mathrm{X}_{\mathrm{a}}\right)$ and $\left(1 / \mathrm{X}^{\prime} \mathrm{a}-1 / \mathrm{X}^{\prime} \mathrm{a}\right)$ are obtained from the element: of the eigonvector matrix ond its inverse. Since the currents camot jump in:jantaneously, Eg.(25) ha:j an adcitional oncillating term

$$
i_{a-o s c i l l a t i n g}-\frac{E_{y}}{\left.x^{\prime \prime}\right]} e^{-\alpha t_{1}} \cos \omega t
$$

$$
+\mathrm{E}_{\mathrm{Q}} \mathrm{e}^{-\alpha t_{\text {ine }}} \mathrm{inwt},
$$

whideh make: $j_{\mathrm{g}} 0$ at $\mathrm{L} 0$. The amplitude of the sine-tern i:s is somal that. it lian be ig nored (equivalent to a reaclance $x_{\text {sine }}$ which is 40 times larger than $x$ "d in the paifer's example with $R_{a}=0$ ).

This osicillatirig term j:i equivalent to the dc offaet in phase guantities, which decays with a time consiant $\mathrm{T}_{3}-1 / \alpha$, with $\alpha$ nearly equal to

$$
\alpha=-\operatorname{\omega R}_{a}\left(\begin{array}{ll}
1 & 1 \\
\mathrm{X}_{\mathrm{d}} & +\mathrm{X}_{\mathrm{q}}
\end{array}\right) .
$$

In the exact solntion, $\alpha \pm j \omega$ is an extra pajr of corjugate complex eigervalues, in addition to the 4 eigenvalues used in the paper.

For $R_{i d}=0$, the osidilating term car be found irdegerdently fyom the two equat ions

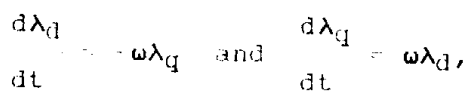

which hats thes solution

$$
\lambda_{\mathrm{d}}=\mathrm{E}_{\mathrm{q}} \cos \omega \mathrm{\omega t} \text { and } \lambda_{\mathrm{q}}=\mathrm{E}_{\mathrm{q}} \operatorname{sin\omega t} \text {. }
$$

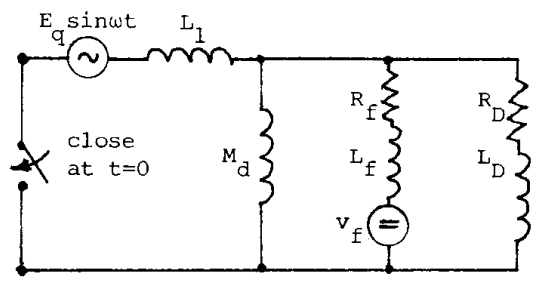

Fig. A. Inirect axiss circuit with sinuevidal voltage jource $F_{i 1}:$ inot.

In the direct axis, this appeario a: a known forcing function in Fig. A, which product:s the iteady-state oscillating term

$$
i_{\text {a o oscillating }}-\frac{E_{1}}{\sqrt{R+X^{2}}} \cos (\omega t \neq 0)
$$

where $R+j X$ is the complex impedance seen by tha voltage source in Fig. $A$, and

$$
\theta-\tan ^{-1}(R / X) \text {. }
$$

This from

impedance can either be calculated

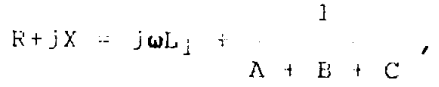

wher: $A: 1 / j \omega M_{1}$,

$$
B-1 /\left(R_{f}+j \omega L_{f} i,\right.
$$

$$
C=1 /\left(R_{D}+j \omega L_{D}\right) \text {, }
$$

or from

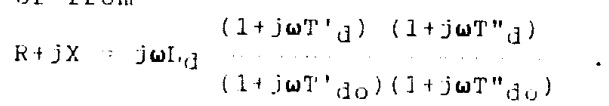

Eq. (V) can be: split intu a cosine cand sine terri,

id-oscillating $-\frac{E_{q}}{\sqrt{R^{2}+X^{2}}} \cos \theta \cos \omega t$

$$
+\frac{E_{Q}}{\sqrt{R^{2}+X^{2}}} \sin \theta \sin \omega t
$$

which, when comparea with Ey. (I), shows that the subtransient reactance obtidined from the exact. sointion is

$$
x{ }_{a}=\frac{\sqrt{R^{2}+x^{2}}}{\cos \theta},
$$

or. with Eq.(VIII),

$$
\begin{aligned}
& \begin{array}{l}
x_{d}+j \frac{x_{d}}{x^{\prime \prime} d+j} x_{s ; n e}
\end{array}
\end{aligned}
$$

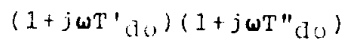

$$
\begin{aligned}
& \left(1+j \omega T^{\prime \prime} a\right)\left(1+j \omega T^{\prime \prime}(a)\right.
\end{aligned}
$$

Using the farameters of Table 5 of the paper for $K_{\text {is }}$. $O$ protuces an $X " d$ froll $E_{1} \cdot(X)$ which is $0.212 \%$ higher than the specified va Iut: of 0.135 E.u. Caray's; data corver:ion

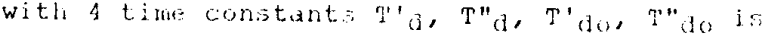




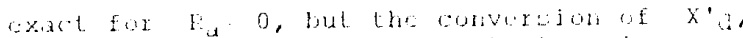

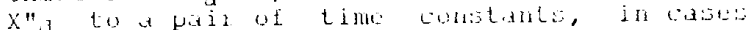
where oniy ont pair is known, is sijghtly in deculate by neylecting $\mathrm{F}_{\mathrm{f}}$ and $\mathrm{F}_{\mathrm{D}}$ in Fig. A. Fromi is practical strmpoint, the inaccuracy of $0.212 \%$ in $x "$, is of cour se negligible.

fior the exate conversion trom $x$ 'a and $x " d$ to a pair of time constint: for $R_{a} 0$, Ec. $\{22 a)$ should be replaced by

$$
\begin{aligned}
& {\left[1+\frac{1}{\left(\omega T^{\prime}{ }_{a}\right)^{2}}\right] \cdot\left(\begin{array}{cc}
1 & 1 \\
L_{1}{ }^{a} & L_{d}
\end{array}\right)}
\end{aligned}
$$

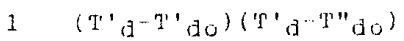

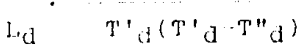

$$
\begin{aligned}
& \text { The reciprocal of the factor } \\
& {\left[1+\frac{1}{\left(\omega T^{1}{ }^{(1}\right)^{2}}\right]}
\end{aligned}
$$

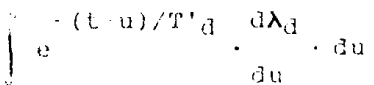

$$
\begin{aligned}
& \left.[]+\frac{1}{\left(\omega \mathrm{T}_{\mathrm{a}}\right)^{2}}\right] \cdot\left(\begin{array}{cc}
1 & 1 \\
\mathrm{I}^{\prime} \mathrm{a} & \mathrm{L},{ }_{\mathrm{a}}
\end{array}\right)
\end{aligned}
$$

in an artalogous way.

In oux Eg.(V).30) of [1] for the definition of hafD, which the authors show in Appen dix A just before paragraph bi, I."a must be replaced by $\mathrm{L}_{\mathrm{d}} \mathrm{T}^{\prime} \mathrm{dT} \mathrm{T}^{\mathrm{d}} /\left(\mathrm{T}^{\prime} \mathrm{du}^{\mathrm{T}}\right.$ "do for the more accurate conversion proposed here.

Canay':; original data conversion is sill ustefus. The errors which it produces in the resistances and inductances are nisuldy neg. ligible from a practical intanjoint. Further more, it profuces injijal guesses for the authors' nethod. Canay's data conversion can also icerve des an indicator of inconistencie: in the masured ata if a negative argument in encounteres in the square root calculation. In such a case. more approximate data comverejons are usially applied.

Manuscript received August 17, 1988.

T. J. Hammons (Glasgow University, Glasgow G12 8QQ, Scotland, U.K.): The authors have provided useful information on the calculation of Synchronous Machine subtransient and transient parameters from sudden short-circuit test by back-solving. They have highlighted unnecessary assumptions and approximations that have been made in the past due in part to the unavailability of measurements and to the cumbersome nature of the calculations needed to remove these approximations. They have described a procedure to remove approximations, and have illustrated how a new philosophy for problem formulation and solution using backsolving programs can simplify and clarify the solution process. In addition, they have introduced a new way of calculating eigenvalues giving decay of the components based on a generalization of the eivenvalue problem.

Investigations performed by this Discussor and reported in Reference [A] indicate that detailed generator models which simulate direct- and quadrature axis sub-transient quantities are necessary for realistic assessments of transient electrical phenomena in large synchronous generators. Significant difference in rotor swing is observed when detailed $(2 \mathrm{~d}, 3 \mathrm{q})$ or reduced (1d, $2 q$ ) and $(1 d, 1 q)$ damper models are used to model the machine's damper circuits. In addition, shaft torque response following a supply system disturbance is noticeably different when detailed or reduced damper models are employed. Damper circuit modelling also has quite a noticeable influence on electrical damping of torsional vibrations in turbine-generator shafts. This is particularly true at low torsional frequencies in addition to that frequency which relates to rotor swing [B]. The influence of subsubtransient quantities [ $(2 \mathrm{~d}$, eq) model] should not be neglected in response torque investigations and in predicting rotor swing.

It should be emphasized that rotor circuit losses which give rise to electrical damping torques are not only in the field and damper windings, but especially in steam turbine generators, mainly in the solid parts of the rotor. It is necessary to consider the losses in the solid iron parts for a realistic and precise computation of electrical damping of rotor swing and torsional vibrations in the machine shaft. This effect is more important for the quadrature-axis due to the absence of the field winding. The effect of solid iron rotor parts is more dominant, for the same reason, in machines without an explicit damper winding. By increasing the number of rotor circuits to three on each axis, i.e., by incorporating the sub-subtransient quantities, the effect of the solid iron parts at subsynchronous frequencies can be considered in a realistic way.

In the case of perturbations in generator rotor speed resulting from rotor swing, and torsional vibration of the shaft when the generator operates on light load, i.e., at a small load angle, electrical damping is effected by currents which flow in the quadrature-axis damper circuits of the synchronous machine. Conversely, at high load angles, direct-axis rotor circuits become the effective mechanism for electrical damping of rotor swing and torsional vibrations in the machine shaft.

It should be noted that detailed governor and avr simulation where gain and phase shift is faithfully modelled at predominant frequencies is also necessary for realistic assessments of rotor swing (and damping of torsional vibrations in turbine-generator shafts). Steam viscous damping acting on turbine rotors should also be simulated in calculating eigenvalues and overall damping of vibrations in turbine-generator shafts. Steam viscous damping acting at each turbine state is assumed to be proportional to respective turbine load. Turbine-generator response following severe disturbances on the system supply is analysed in References $[A],[B]$ and [C].

Figure A illustrates the effect damper circuit modelling (and the fault clearing process) has on rotor swing following a $185 \mathrm{~ms} \mathrm{~L}-\mathrm{L}-\mathrm{L}$ non-ground HV system short-circuit with clearance. The responses relate to a $500 \mathrm{MW}$, $3600 \mathrm{rpm}$ machine. Automatic voltage regulator (avr) and governor action

\begin{tabular}{|c|c|c|c|c|}
\hline Model 1: & $\begin{array}{l}x_{d}=1.77 \\
x_{c}=0.0825 \\
x_{q}=1.65\end{array}$ & $\begin{array}{l}x_{d}^{\prime}=0.311 \\
T_{d}^{\prime}=0.816 \\
x_{q}^{\prime}=0.767 \\
T_{q}^{\prime}=0.226\end{array}$ & $\begin{array}{l}x_{d}^{\prime \prime}=0.274 \\
T_{d}^{\prime \prime}=0.069 \\
x_{q}^{\prime \prime}=0.424 \\
T_{q}^{\prime \prime}=0.0314\end{array}$ & $\begin{array}{l}x_{d}^{\prime \prime \prime}=0.221 \\
T_{d}^{\prime \prime \prime}=0.0031 \\
x_{q}^{\prime \prime \prime}=0.240 \\
T_{q}^{\prime \prime \prime}=0.0024\end{array}$ \\
\hline Model 2: & $\begin{array}{l}x_{d}^{\prime}=0.301 \\
T_{d}^{\prime}=0.77\end{array}$ & $\begin{array}{l}x_{d}^{\prime \prime}=0.256 \\
T_{d}^{\prime \prime}=0.022\end{array}$ & $\begin{array}{l}x_{q}^{\prime}=0.62 \\
T_{q}^{\prime}=0.140\end{array}$ & $\begin{aligned} x_{q}^{\prime \prime} & =0.322 \\
T_{q}^{\prime \prime} & =0.011\end{aligned}$ \\
\hline Model 3: & $\begin{array}{l}x_{d}^{\prime}=0.301 \\
T_{d}^{\prime}=0.77\end{array}$ & $\begin{array}{l}x_{d}^{\prime \prime}=0.256 \\
T_{d}^{\prime \prime \prime}=0.022\end{array}$ & $\begin{array}{l}x_{q}^{\prime \prime}=0.322 \\
T_{q}^{\prime \prime}=0.011\end{array}$ & \\
\hline
\end{tabular}
has been ignored.

Curves (i)-(iii) depict that rotor acceleration is less following fault incidence when the detailed ( $2 \mathrm{~d}, 3 \mathrm{q})$ damper model is employed. Peak rotor angle to effective infinite busbar corresponding to the $(2 d, 3 q),(1 d, 2 q)$ and (1d, lq) damper models for fault clearance at fault current zeros where supply system infeed is simulated is $92.6^{\circ}, 99.0^{\circ}$ and $97.0^{\circ}$ respectively Peak rotor angle corresponding to the detailed ( $2 \mathrm{~d}, 3 \mathrm{q})$ damper representation following simultaneous fault clearance is $91.1^{\circ}$. Peak rotor angle is significantly less when the detailed representation is employed on accoun of improved low-frequency electrical damping of rotor swing. Peak rotor

Table A. Time Constants Determined from Frequency Response Loci for 500MW, 3600 r.p.m. Machine 


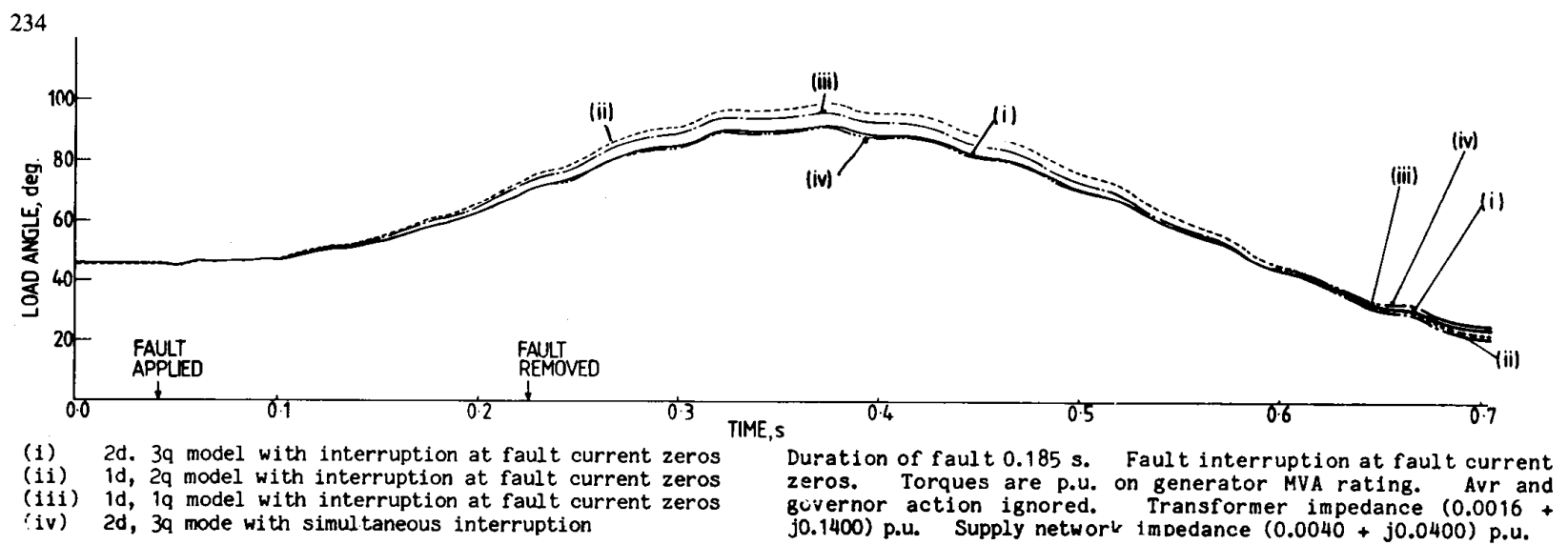

Figure A. Effect of Damper Modelling on Rotor Angle following L-L-L non-ground HV System Disturbance, 500MW, 3600 r.p.m. Machine

angle is marginally lower following simultaneous interruption on account of reduced effective duration of the fault.

For the machine model, three, two and one rotor circuit fits to standstill frequency response (SFR) test data (or equivalent data computed by finiteelement analysis) as depicted by the equivalent circuits of Figure 2 of Reference [A] were made. The fits were then corrected as appropriate for the low flux level of the SFR tests through a comparison of the open-circuit test airgap line gradient with that obtained from measurement of the statorrotor transfer function at very low frequency. It should be noted that all three models yield nearly the same short-circuit behaviour (the deviations are negligible). This is on account of the sub-subtransient time constant of the $\mathrm{d}$-axis $(0.0031 \mathrm{~s})$ being very small, so that the main portion of

$$
V_{q}\left[1 / x_{d^{*}}-1 / x_{d^{*}}\right] \exp \left(-t / T_{d^{*}}\right)
$$

is not visible in the oscillographic record of the phase current following a sudden short-circuit. Time constants and reactances for the three models are given in Table A. Determination of Synchronous Machine parameters from characteristic quantities applicable to sub-subtransient data is discussed in Reference [D].

It would seem from the above that detailed generator models which simulate direct- and quadrature-axis sub-subtransient phenomena are essential for predicting generator transient behaviour following a severe supply-system event, such as rotor swing and shaft torsional response. It would appear that this phenomena has not been represented in the analyses the authors have made. Could the authors' method be extended to include determination of synchronous machine sub-subtransient parameters, in addition to subtransient and transient parameters, from sudden short-circuit tests by back-solving? While generalization of the eigenvalue problem along with the use of a back solver permits the removal of approximations for a (1d, $2 q$ ) subtransient model during machine parameter determination, such as neglect of $R_{a}$, sub-subtransient components may decay too fast for the procedure to be adapted to give realistic sub-subtransient constants for the machine. The authors' views on this Discussor's observations would enhance usefuleness of the presented results.

\section{References}

[A] T. J. Hammons and I. M. Canay. "Effect of Damper Modelling and the Fault Clearing Process on Response Torque and Stressing of Turbine-Generator Shafts", IEEE Transactions on Energy Conversion, Vol. EC-1, (1), 1986, pp. 113-121.

[B] T. J. Hammons. "Effect of Fault Clearing and Damper Modelling on Excitation and Decay of Vibrations in Generator Shafts following Severe Disturbances on the System Supply", IEEE Transactions on Energy Conversion, Vol. EC-2, (2), 1987, pp. 308-320.

[C] T. J. Hammons, M. Istin and A. Crocquevielle. "Analysis of Continuum and Reduced Shaft Models in Evaluating TurbineGenerator Shaft Torsional Response following Severe Disturbances on the System Supply'. Electric Machines and Power Systems, Vol. 13, (6), 1987, pp. 387-408.

[D] I. M. Canay. "Determination of Synchronous Machine Model Parameters from Characteristic Quantities Applicable also to SubSubtransient Data'. Electric Machines and Power Systems, Vol. 9, (1), 1984, pp. 33-48

Manuscript received August 4, 1988.

\section{FERNANDO L. ALVARADO AND CLAUDIO CAÑIZARES}

The authors wish to thank the discussers for their interest in our paper. We respond to Professor Dommel's comment first. Professor Dommel discusses a slight inaccuracy in the calculation of the parameters. The effect of this inaccuracy is that it is not possible to exactly reproduce the "measured" data with the calculated parameters. The inaccuracy occurs whether the "backsolving" procedure advocated in the paper or any other calculation procedure is used. The reason for this error is attributed to the effect of the field and damper winding resistance on the sudden-short circuit currents. We agree with his comment, and wish to take a slightly different viewpoint more consistent with our backsolving approach.

The problem is due in part to the incomplete nature of the definition of sudden short currents used in the standard tests. The standard definition (IEEE standard 1151983) of the sudden-short circuit current for the d axis that we reproduce here is shown in equation (25) in the paper:

$i_{d}=\frac{E_{q}}{x_{d}}+E_{q}\left(\frac{1}{x_{d}{ }^{\prime}}-\frac{1}{x_{d}}\right) e^{-t / T_{d}^{\prime}}+E_{q}\left(\frac{1}{x_{d} "}-\frac{1}{x_{d}^{\prime}}\right) e^{-t / T_{d} "}$

This definition neglects the armature resistance $R_{a}$. It also neglects the sinusoidal oscillation of the field current and considers only its average effect. To take into account the complete solution of the sudden-short circuit current for this specific case, another term should be added. This additional term would have to be the oscillating term that comes from the phasor solution of the machine equations due to the flux $\lambda_{d}$. In addition to equations (1) in the paper, the complete differential equations describing machine behavior include additional differential equations for $\lambda_{d}$ and $\lambda_{q}$ as follows:

$$
\frac{d \lambda_{d}}{d t}=-v_{d}-R_{a} i_{d}-\omega \lambda_{q} \quad \frac{d \lambda_{q}}{d t}=-v_{q}-R_{a} i_{q}+\omega \lambda_{d}
$$

Under short circuit conditions both $v_{d}$ and $v_{q}$ are zero. If we neglect $R_{a}$ these equations reduce to:

$$
\frac{d \lambda_{d}}{d t}=-\omega \lambda_{\mathrm{q}} \quad \frac{\mathrm{d} \lambda_{\mathrm{r}}}{\mathrm{dt}}=\omega \lambda_{\mathrm{d}}
$$

If we solve these equations for no-load initial conditions then:

$$
\lambda_{d}=-\frac{E_{\alpha}}{\omega} \cos (\omega t) \quad \lambda_{q}=-\frac{E_{q}}{\omega} \sin (\omega t)
$$

The average value for both these terms is zero. If the armature resistance had been considered, these fluxes would eventually decay to zero amplitude as well. It is 
possible to solve these armature equations separately from the remaining rotor circuit equations. We now consider the effect of these armature fluxes on the currents:

$$
\begin{gathered}
\frac{d \lambda_{f}}{d t}=-R_{f} i_{f}-v_{f} \\
\frac{d \lambda_{D}}{d t}=-R_{D} i_{f} \\
\omega \lambda_{d}=x_{d} i_{d}+x_{M d} i_{f}+x_{M d} i_{D} \\
\omega \lambda_{f}=x_{M d} i_{d}+x_{f f} i_{f}+x_{M d} i_{D} \\
\omega \lambda_{D}=x_{M d} i_{d}+x_{M d} i_{f}+x_{D D} i_{D}
\end{gathered}
$$

Because we have already determined $v_{d}$, we can treat this problem as having two forcing functions, namely: $v_{f}$ (DC) and $\lambda_{d}(\mathrm{AC})$. We then use superposition to find a steady state solution for $i_{\alpha}$. The DC term $c$ an be calculated by setting the derivatives $(d / d t)$ and the cosine forcing function $\left(\lambda_{d}\right)$ equal to zero, obtaining:

$$
i_{d D C}=\frac{E_{q}}{x_{d}}
$$

which agrees with the definition in the standards. For the AC term, the DC forcing function $\left(v_{f}\right)$ has to be set equal to zero and the derivative term $(d / d t)$ has to be replaced by $j \omega$. This produces a set of phasor equations. In this case the phasor form of the forcing function is:

$$
\tau_{d}=-\frac{E_{q}}{\omega}
$$

The analytic solution to this set of phasor equations is:

$$
\begin{gathered}
\tilde{I}_{d}=-\frac{E_{d}}{x_{d}}\{1+ \\
\left.\frac{j \frac{x_{M d}^{2}}{x_{d}}\left[R_{f}+R_{D}+j\left(x_{f f}+x_{D D}-2 x_{M d}\right)\right]}{\left(R_{f}+j x_{f f}-j \frac{x_{M d}^{2}}{x_{d}}\right)\left(R_{D}+j x_{D D}-j \frac{x_{M d}}{x_{d}}\right)+\left(x_{M d}-\frac{x_{M d}}{x_{d}}\right)^{2}}\right\} \\
\check{I}_{d}=-\frac{E_{d}}{z_{e q}} e^{j(\omega \tau+\phi)}
\end{gathered}
$$

In the time domain this solution produces the oscillating term:

$$
i_{\mathrm{dosC}_{1}}=-\frac{E_{q}}{z_{\text {eq }}} \cos (\omega t+\phi)
$$

If this term is added to the standard definition for $i_{d}$ the equation is not entirely consistent. In particular, the initial current is not zero. What it has been usually done to avoid this problem is to define the oscillating term as:

$$
i_{d_{\mathrm{OSC}_{2}}} \approx-\frac{E_{\mathrm{q}}}{x_{d}{ }^{\prime \prime}} \cos (\omega t)
$$

In this case $x_{d}$ " is defined as the equivalent of the parallel reactances in the star circuit model of the machine, which is what we get if $R_{f}$ and $R_{D}$ are neglected in equation ( $f$ )

For the IEEE benchmark model of the machine used in the paper, the difference between the two possible magnitudes of the oscillating term can be calculated using the SOLVER-Q complex setup shown below. This produces a calculated subtransient impedance $\left(x_{d} "\right) 0.2118 \%$ off the specified value. This agrees with Dr. Dommel's calculation.

\section{Machine equations}

$11 *_{w} *_{\text {lfss }}=-r f \star_{\text {ifss }}$ :

$11 * w * 1 d p s s=-r d p * i d p s s$

xd * idss + xmd* ifss + xmd * idpss $=$ eq

xmd idss $+x f$ * ifss + xnd * idpss $=w \star$ lfss

xmd $*$ idss + xnd $*$ ifss $+x d p *$ idpss $=w *$ ldpss ;
The small discrepancy between these two values obtained for the oscillating part of the sudden-short circuit current does not appear in any other of the terms that form part of $i_{d}$. For these terms, the eigenvalue analysis of the decoupled problem gives the same values as the ones obtained from the definition in the standards. This suggests that equations $(22 \mathrm{a})$ and $(22 \mathrm{~b})$, which are based on the definition of $\mathrm{x}_{\mathrm{d}} "$ as the parallel of the reactances in the star circuit, are in slight error. These equations are also used by the Canay procedure. H. Dommel outlines a procedure to eliminate this approximation. We consider an alternative. The error can be avoided by using the open circuit time constants directly, together with $X_{d}$ and $X_{d}$ ". To do this the following equations have to be added to the analysis:

$$
\begin{aligned}
& T_{d o}{ }^{\prime}+T_{d o}{ }^{\prime}=\frac{L_{f f}}{R_{f}}+\frac{L_{D D}}{R_{D}} \\
& T_{d o} T_{d o}^{\prime}=\frac{L_{f f} L_{D D}-M_{d}{ }^{2}}{R_{f} R_{D}}
\end{aligned}
$$

These equations come directly from the eigenvalue analysis of the open circuit machine equations, where $R_{a}$ and the approximations on the transient and subtransient reactances do not play any role $\left(i_{d}\right.$ and $i_{q}$ are zero).

A second cause of slight error comes from the assumption that the sinusoidal terms of the field and damper winding fluxes do not influence the determination of the initial conditions, also a consequence of neglecting the resistances $R_{f}$ and $R_{D}$ in the definition of $x_{d}$. . This error can be suppressed by adding to our problem the set of equations shown above in the SOLVER-Q setup for the AC steady-state analysis $\left(R_{a}=0\right)$. The equations used to determine the scaling factors for the eigenvectors have to be replaced by equations (j) that include these oscillating terms at $t=0$.

$$
\begin{gathered}
\lambda_{\mathrm{f}_{0}}-\lambda_{\mathrm{fDC}_{\mathrm{DC}}}=\alpha_{1} \lambda_{\mathrm{f}_{1}}+\alpha_{2} \lambda_{\mathrm{f}_{2}}+\operatorname{Re}\left\{\tilde{\lambda}_{\mathrm{f}_{\mathrm{AC}}}\right\} \\
\lambda_{\mathrm{DD}_{\mathrm{D}}}-\lambda_{\mathrm{DD} \mathrm{DC}}=\alpha_{1} \lambda_{\mathrm{DD}_{1}}+\alpha_{2} \lambda_{\mathrm{DD}_{2}}+\operatorname{Re}\left\{\tilde{\lambda}_{\mathrm{DD}_{\mathrm{AC}}}\right\}
\end{gathered}
$$

These implicit equations can be used without any further manipulation directly into a SOLVER-Q setup. The solution to this problem for the $d$ axis gives slightly different results from the ones shown in the paper for the two winding machine model:

$$
\begin{array}{ll}
R_{f}=0.00140679 & x_{f}=1.72178915 \\
R_{D}=0.00406988 & x_{D}=1.66545811
\end{array}
$$

Not mentioned by either discusser or in the paper is perhaps a greater potential source of error. The standard definition neglects the possible existence of quadrature axis terms in the direct axis sudden-short current. However, the presence of $R_{a}$ may require the consideration of a more complete form for this current if sudden-short circuit tests are to be used:

$\dot{i}_{d}=\beta_{1}+\beta_{2} e^{-t / T_{d^{\prime}}}+\beta_{3} e^{-t / T_{d^{\prime \prime}}}+\beta_{4} e^{-t / T_{q^{\prime}}}+\beta_{5} e^{-t / T_{q}{ }^{\prime \prime}}+\beta_{6} e^{-t / T_{a}} \cos (\omega t+\phi)$

As a result, we believe that a more accurate way of calculating the circuit parameters can be achieved by using the results of the open circuit tests, where the coupling between axis does not exist.

Professor Hammons starts his discussion with an excellent overview of the contributions of our paper. We could not have stated them better ourselves. Then he proceeds to discuss the merits of considering a subsubtransient winding (probably due to eddy current effects) on machine transients. Introducing a third winding in the simulation of the synchronous machine yields a model closer to reality, specially for the analysis of torques and rotor swings. $\mathrm{He}$ then asks whether the proposed procedure would be capable of producing 
reasonable circuit parameters for such model. We show in this closure that the answer is yes. We created a SOLVER-Q setup that included the additional windings for the $d$-axis. The transient, subtransient and sub-subtransient parameters were those given by Hammons. Just a few changes had to be made to the original file shown in the paper to include into the simulation the third winding. We calculated initial guesses using the Canay procedure. Good initial guesses proved essential for good convergence. These were obtained from the two winding model.

The results obtained (for $R_{a}=0$ ) are as follows:

$$
\begin{aligned}
& R_{f}=0.001127819 \\
& R_{D}=0.045878795 \\
& R_{D D}=0.474653206 \\
& \mathrm{R}_{\mathrm{g}}=0.015743180 \\
& \mathrm{R}_{\mathrm{Q}}=0.064774170 \\
& \mathrm{R}_{Q Q}=0.391118670 \\
& x_{f}=1.955267642 \\
& \mathrm{X}_{\mathrm{D}}=2.821476343 \\
& \mathrm{X}_{\mathrm{DD}}=2.184718014 \\
& \mathrm{x}_{\mathrm{g}}=2.828957507 \\
& x_{0}=2.259965955 \\
& \mathrm{X}_{Q Q}=1.855319504
\end{aligned}
$$

We regard these values as reasonable, based on the set of measurements available to us and on the values calculated with Canay's equations from the set of data for the two winding model, which are shown below.

$$
\begin{array}{ll}
R_{f}=0.0011408 & x_{f}=1.9398053 \\
R_{D}=0.1070040 & x_{D}=2.5155586 \\
R_{g}=0.0174220 & x_{Q}=2.4075808 \\
R_{Q}=0.1198928 & x_{Q}=1.9935677
\end{array}
$$

The inclusion of $R_{a}$ into the determination of the parameters should not be a problem. In this case the $d$ and $q$ axes would be considered together, and with good initial guesses obtained from the decoupled analysis, the solution would be straight forward.

We conclude by stating that the even with a tool such as SOLVER-Q, we encountered convergence difficulties. Very good initial guesses (obtained from classic methods) were required in some cases. We look forward to improved versions of this type of software that will have even more robust global convergence characteristics.

Manuscript received September 19, 1988. 